\title{
Infecciones por Listeria monocytogenes en mujeres embarazadas: experiencia del Hospital Clínico de la Pontificia Universidad Católica de Chile
}

\author{
Demetrio Larraín de la C., Fernando Abarzúa C., Francisca de Jourdan H., Paulina Merino O., \\ Cristián Belmar J. y Patricia García C.
}

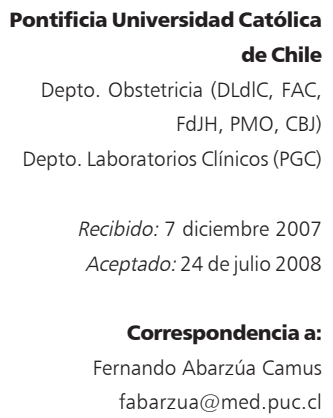

Pontificia Universidad Católica Depto. Obstetricia (DLdIC, FAC, $\mathrm{FdJH}, \mathrm{PMO}, \mathrm{CBJ})$ (tabarzua@med.puc.cl

\section{Listeria monocytogenes infection in pregnancy: experience of Pontificia Universidad Católica de Chile University Hospital}

Listeria monocytogenes is Gram-positive facultative intracellular pathogen often foodborne and found elsewhere. It is an uncommon cause of illness in the general population. However, it is an important cause of severe infection in neonates, pregnant women, elderly and immunosuppressed patients. Listeriosis has unique preference for pregnant women. Maternal listeriosis is a diagnostic challenge, and intrauterine infection can lead to severe complications such as amnionitis, preterm labor, spontaneous abortion, stillbirth and neonatal sepsis. From 2001 to 2005, 16 patients with L. monocytogenes were identified in this hospital; four $(25 \%)$ were pregnant women. Clinical and laboratory findings are described. There were 3 preterm deliveries and 1 spontaneous second trimester abortion. Three women with listeriosis had no predisposing factors other than pregnancy. One patient was on immunosupressive drugs for ulcerative colitis. Fever was the most common symptom. Infected neonates were most commonly diagnosed with early-onset listeriosis (two cases) or fetal demise (one case). Pregnancy can be the only risk factor for listeriosis. Listeriosis should be considered during the evaluation of febrile syndrome in pregnancy as this condition can be the only risk factor. Blood and amniotic fluid cultures are useful diagnostic tests. Perinatal complications remains high.

Key words: Listeria monocytogenes, listeriosis, meningitis, pregnancy, fetal demise.

Palabras clave: Listeria monocytogenes, listeriosis, meningitis, embarazo, óbito fetal.

\section{Introducción}

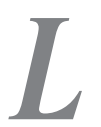

isteria monocytogenes, agente infeccioso causal de la listeriosis, es un bacilo grampositivo que infecta a humanos y animales. Ha sido reconocido como patógeno en seres humanos por más de 70 años, afectando principalmente a mujeres embarazadas, recién nacidos (RNs), ancianos, pacientes diabéticos, cirróticos, oncológicos, insuficientes renales e inmunocomprometidos, en general. Su principal vía de contagio es a través de la ingestión de agua y alimentos contaminados ${ }^{1,2}$. Este microorganismo se encuentra ampliamente distribuido en la naturaleza y cadena alimentaria, habita en múltiples nichos ecológicos (polvo, agua, suelo, vegetales, carne, leche y sus derivados, crustáceos, peces, insectos, etc) y forma parte de la microbiota fecal de muchos mamíferos, aves ${ }^{3} \mathrm{y} \sim 10 \%$ de la población humana ${ }^{4}$.

Las manifestaciones clínicas de la listeriosis pueden variar y diferir sustancialmente entre los distintos grupos. En sujetos previamente sanos, suele manifes- tarse como una gastroenteritis aguda febril, generalmente leve y autolimitada ${ }^{5}$. En individuos inmunocomprometidos, en cambio, puede desencadenar cuadros de mayor gravedad y alta mortalidad como meningitis, meningoencefalitis, encefalitis (rombencefalitis), absceso cerebral o septicemia ${ }^{5-7}$.

El riesgo de infección es mayor durante el embara$\mathrm{ZO}^{8,9}$. La mayoría de las mujeres embarazadas permanece asintomática o experimenta síntomas inespecíficos compatibles con un estado gripal (flu-like illness), caracterizado por mialgias, cefalea, fiebre y compromiso del estado general. La asociación de síntomas gastrointestinales como diarrea y cólicos abdominales es menos frecuente ${ }^{1}$. El compromiso del SNC es inusual en pacientes embarazadas, existiendo escasos reportes de meningitis por L. monocytogenes durante la gestación ${ }^{10,11}$. La listeriosis puede producir aborto espontáneo, restricción de crecimiento intrauterino y desencadenar trabajo de parto prematuro, corioamnionitis clínica y óbito fetal ${ }^{5,8,12,13}$.

El objetivo de este trabajo es presentar la experien- 


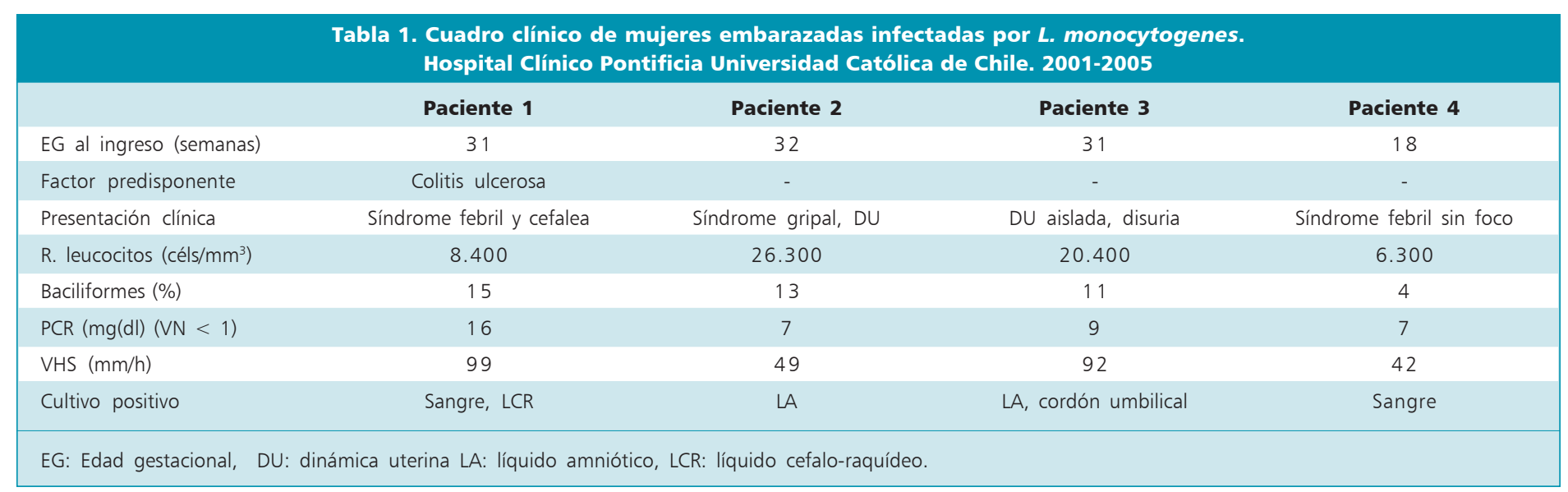

cia del Hospital Clínico de la Pontificia Universidad Católica de Chile en el diagnóstico y tratamiento de la infección por L. monocytogenes en mujeres embarazadas, entre los años 2001 y 2005. Dada la baja frecuencia de la listeriosis perinatal, se presenta una revisión del tema basada en la evidencia disponible en la literatura, a fin de dar algunas pautas para el manejo de esta entidad.

\section{Pacientes y Método}

Se analizaron retrospectivamente los registros del laboratorio de microbiología del Hospital Clínico de la Pontificia Universidad Católica de Chile, entre los años 2001 y 2005. Se seleccionaron aquellos casos compatibles con listeriosis perinatal (hemocultivos y/o cultivos de LCR en mujeres embarazadas, cultivos de líquido amniótico o cultivos de cordón umbilical) que resultaron positivos para L. monocytogenes. Se revisaron las fichas de estas pacientes y sus $\mathrm{RNs}$, recolectando datos con respecto a edad materna, patologías de base, control prenatal, motivo de consulta, edad gestacional, estudios de laboratorio, vía de parto, tratamiento materno y fetal, resultado perinatal, seguimiento del $\mathrm{RN}$ y hallazgos anátomo-patológicos en el feto y/o placenta según corresponda. Se describe brevemente cada uno de los casos.

\section{Resultados}

Durante el período analizado, se aisló L. monocytogenes en un total de 22 muestras: 16 hemocultivos, dos cultivos de líquido amniótico, dos cultivos de LCR, un cultivo de secreción ocular neonatal y un cultivo de secreción de cordón umbilical, correspondientes a 15 pacientes. Sólo cuatro pacientes $(25 \%)$ eran mujeres embarazadas. Los dos cultivos positivos
Tabla 2. Cuadro clínico en RN de madres infectadas por L. monocytogenes

\begin{tabular}{|c|c|c|c|c|}
\hline & RN 1 & RN 2 & RN 3 & RN 4 \\
\hline EG (semanas) & 31 & 32 & 31 & 18 \\
\hline PN (gr) & 1.610 & 2.185 & 1.910 & 290 \\
\hline Causa de la interrupción & $\begin{array}{c}\text { Sufrimiento } \\
\text { fetal }\end{array}$ & Corioamnionitis & Corioamnionitis & $\begin{array}{l}\text { Óbito } \\
\text { fetal }\end{array}$ \\
\hline Infección perinatal & No & Sí & Sí & Sí \\
\hline Cultivos positivos & - & Secreción ocular & Cordón umbilical & - \\
\hline
\end{tabular}

en $\mathrm{RN}(13 \%)$ correspondían a hijos de las pacientes infectadas y se consideraron como caso único. A continuación se describen, brevemente, los cuatro casos. El cuadro clínico y principales hallazgos de laboratorio maternos se resumen en la Tabla 1; y los de los fetos y/o RN, en la Tabla 2.

Caso $N^{o} 1$. Paciente de 23 años, nulípara, portadora de colitis ulcerosa diagnosticada un mes previo al embarazo, en tratamiento con prednisona $30 \mathrm{mg} /$ día y mesalazina $4 \mathrm{~g}$ /día. A las 27 semanas de embarazo fue hospitalizada y tratada por cuadro de colitis pseudomembranosa por Clostridium difficile, que evolucionó favorablemente. Fue internada en nuestro centro a las 32 semanas de gestación con cefalea holocránea intensa, mialgias y fiebre, evolución de seis días. Dado el cuadro clínico se tomaron hemocultivos y realizó una punción lumbar. Se descartó infección ovular con amniocentesis y constató el bienestar fetal, por lo que se decidió observar una conducta expectante. Se aisló L. monocytogenes en sangre y LCR, iniciando tratamiento endovenoso con ampicilina $12 \mathrm{~g}$ /día y se indujo madurez pulmonar fetal con corticosteroides. Al 
tercer día de tratamiento el monitoreo fetal fue sospechoso de sufrimiento fetal, por lo que se decidió interrumpir el embarazo. Se le realizó una operación cesárea extrayéndose un RN de pretérmino, con 1.610 gramos de peso, Apgar 6-9. El RN no presentó signos clínicos ni de laboratorio compatibles con infección, recibió antimicrobianos profilácticos y evolucionó en forma favorable sin secuelas. Los hemocultivos resultaron negativos. La madre completó 28 días de tratamiento con ampicilina, evolucionando favorablemente.

Caso $N^{o}$ 2. Paciente de 33 años, nulípara, sin antecedentes mórbidos. Consultó a las 32 semanas de gestación por un cuadro caracterizado por fiebre, mialgias, cefalea y compromiso del estado general (flu-like illness), asociado a dinámica uterina intermitente, evolución de dos semanas. Ingresó con el diagnóstico de trabajo de parto prematuro febril. Se le realizó una amniocentesis, obteniéndose líquido amniótico con meconio fluido y exámenes citoquímicos compatibles con infección intra-amniótica. La tinción de Gram directa visualizó bacilos grampositivos, por lo que se inició tratamiento bi-asociado con ampicilina y gentamicina y se decidió interrumpir el embarazo. Los hemocultivos maternos resultaron negativos. El cultivo de líquido amniótico fue positivo para L. monocytogenes. Durante el trabajo de parto se presentó un registro fetal no tranquilizador por lo que se realizó una cesárea de urgencia. Se obtuvo un RN pretérmino de 2.185 gramos, deprimido, Apgar 5-7 que requirió reanimación, intubación, ventilación mecánica y manejo en UCI. Se aisló L. monocytogenes desde la secreción ocular. Los hemocultivo y cultivo de LCR del RN fueron negativos. Evolucionó con shock séptico grave, enfermedad de membrana hialina, hipertensión pulmonar persistente y bronconeumonía connatal. Estuvo 36 días hospitalizado y se fue de alta en buenas condiciones generales. La madre evolucionó favorablemente.

Caso $N^{\circ}$ 3. Paciente de 17 años, nulípara, sin antecedentes mórbidos. Ingresó a las 31 semanas de gestación por trabajo de parto prematuro. Al ingreso se le practicó una amniocentesis obteniéndose líquido amniótico con meconio y estudio citoquímico sugerente de infección intra-amniótica. Se tomó cultivo del líquido amniótico y se inició tratamiento con un esquema bi-asociado ampicilina y gentamicina. El trabajo de parto fue acelerado con oxitocina, resolviéndose por vía vaginal. Nació un niño de pretérmino, con 1.910 gramos de peso, hipotónico y bradicárdico (Apgar 16-8). Requirió reanimación, intubación y manejo en UCI. Completó 10 días de tratamiento con ampicilina y gentamicina, evolucionando favorablemente. Se aisló
L. monocytogenes en el líquido amniótico y en una muestra de secreción de cordón umbilical.

Caso $N^{o}$ 4. Paciente de 39 años, multípara de 2, sin antecedentes mórbidos. Ingresó a las 18 semanas de embarazo con un síndrome febril sin foco, con evolución de cuatro días. La ecografía obstétrica de ingreso resultó normal. Se le tomaron cultivos de sangre y orina e inició tratamiento antimicrobiano bi-asociado con ampicilina y gentamicina. Hubo desarrollo de $L$. monocytogenes en los hemocultivos. Al tercer día de tratamiento se constató ausencia de latidos cardiofetales. El trabajo de aborto se inició en forma espontánea ese mismo día. En la biopsia se informó la existencia de una corioamnionitis purulenta con microabscesos placentarios. El feto, de aproximadamente 20 semanas, tenía bronquiolitis necrosante exudativa, bronconeumonía acinosa y meningitis linfohistiocitaria. La evolución materna fue favorable. Completó tres semanas de antibioterapia.

\section{Discusión}

Listeria monocytogenes es un bacilo grampositivo, intracelular facultativo, $\beta$-hemolítico, anaerobio facultativo, no esporulado y móvil. Mide 0,5 x $1,5 \mu \mathrm{m}$ y es capaz de sobrevivir bajo condiciones extremas de $\mathrm{pH}$, salinidad y temperatura, pudiendo desarrollarse sin problemas a temperaturas de refrigeración $\left(-18^{\circ}-10^{\circ} \mathrm{C}\right)$, por lo que puede ser transmitida a través de alimentos adecuadamente refrigerados, incluso congelados ${ }^{14}$. Listeria monocytogenes es destruida a través de la pasteurización y por la mayoría de los agentes desinfectantes ${ }^{15}$. Se aisla fácilmente, a partir de sitios normalmente estériles, mediante el uso de cultivos corrientes; no obstante, en cultivos mixtos o polimicrobianos como coprocultivos o alimentos, es más difícil, por lo que requiere el uso de medios enriquecidos o selecti$\operatorname{vos}^{1}$.

Se han identificado seis especies de Listeria, pero L. monocytogenes, es la principal patógena en humanos y animales. Existen descritos 16 serotipos de Listeria monocytogenes, según sus antígenos de superficie; sin embargo, sólo 3 serotipos $(1 / 2 \mathrm{a}, 1 / 2 \mathrm{~b}, \mathrm{y}$ 4b) son responsables de $95 \%$ de las infecciones en humanos ${ }^{2,16-18}$; su serotipificación es útil desde el punto de vista epidemiológico más que clínico.

La puerta de entrada es el tracto digestivo y $L$. monocytogenes es capaz de tolerar el pH gástrico, enzimas proteolíticas y sales biliares, gracias a la traducción de proteínas de estrés ${ }^{19}$. Además, es capaz de inducir su captación por las células del hospedero y de esta forma, la bacteria es traspasada de célula a célula, pudiendo vivir por largo tiempo en el interior de 
macrófagos y células no fagocíticas del hospedero. Después de esta fase epitelial, L. monocytogenes alcanza el torrente sanguíneo, teniendo la capacidad de traspasar tres importantes barreras: intestinal, hematoencefálica y placentaria. Este enteropatógeno tiene especial tropismo por la placenta y el SNC. La infección transplacentaria y del SNC se debería a la interacción especie-específica de la internalina (bacteriana) y el receptor E-cadherina en la vellosidad coriónica y, probablemente, en las meninges ${ }^{20}$.

La listeriosis puede ocurrir en forma epidémica, causando brotes de enfermedad a través de la ingestión de alimentos contaminados, o esporádica, modalidad mucho más frecuente y cuya vía de transmisión no es del todo entendida ${ }^{21,22}$. En nuestra experiencia, todos fueron casos esporádicos $\mathrm{y}$, si bien se preguntó dirigidamente en relación a posibles fuentes de contagio, éstas no fueron identificadas. En 1981, la investigación de un brote de listeriosis en Nova Scotia, Canadá, puso en evidencia la transmisión de L. monocytogenes a través de los alimentos (repollo), por primera vez. Se reportaron además 34 casos de infección perinatal, con 19 óbitos y mortalidad perinatal de $27 \%^{23}$. Desde entonces se han descrito otros brotes, todos con una mortalidad perinatal cercana a $30 \%^{24,25}$. Los alimentos involucrados son variados e incluyen cecinas, embutidos, salchichas, quesos blandos, mantequilla, leche no pasteurizada, pescados ahumados, vegetales, etc $^{21,26}$. No existe un patrón estacional claro, aunque la mayoría de las infecciones reportadas ocurren durante el verano ${ }^{27-29}$.

Se estima una incidencia universal anual de 0,2 a 5,6 casos por millón de habitantes, con una mortalidad global que oscila entre 18 y $50 \%$, según los distintos reportes $^{27,17,29}$. La listeriosis perinatal representa entre 13 y $43 \%$ de los casos, con una alta mortalidad perinatal $(34 \text { a } 56 \%)^{27,28}$. Una revisión de 178 casos acaecidos durante el embarazo, publicada en 2002, mostró que en $20 \%$ de ellos se producía muerte fetal y de $80 \%$ que nacía vivo, $68 \%$ estaba infectado (24\% moría en el período post-natal y $12 \%$ presentaba secuelas neurológicas). Sólo $31 \%$ nacía sin infección, y su evolución post-natal era dependiente de la edad gestacional de nacimiento ${ }^{8}$. En nuestra serie, en el caso $\mathrm{N}^{0} 1$ se descartó la infección intra-amniótica mediante amniocentesis y los hemocultivos del RN fueron negativos. En los otros tres casos (75\%) hubo compromiso fetoneonatal; uno de ellos con shock séptico y otro con óbito fetal durante el segundo trimestre.

El espectro clínico de las infecciones por este agente es extremadamente amplio, desde cuadros leves y autolimitados, en sujetos sanos, hasta cuadros graves con shock séptico, falla multiorgánica y muerte. La meningitis, meningo-encefalitis y sepsis son las for- mas de presentación clínica más frecuentes en adultos inmunocomprometidos 5 . Por otra parte, L. monocytogenes, es causa muy infrecuente de meningitis en mujeres adultas embarazadas. Existen menos de cinco casos reportados en la literatura internacional y el caso $\mathrm{N}^{\circ} 1$ de nuestra serie sería el primero reportado en nuestro país ${ }^{8,28,30-32}$.

La infección neonatal se divide esquemáticamente en listeriosis neonatal precoz y tardía. La primera se presenta en los primeros cuatro días de vida, frecuentemente en prematuros, quienes adquieren el microorganismo por vía transplacentaria y en $50 \%$ de los casos desarrollan septicemia, con o sin compromiso meníngeo. Es la forma más frecuente de ver en obstetricia. Como se mencionó, tres de los cuatro fetos/RN de nuestra serie presentaron compromiso infeccioso grave, pero no se aisló el agente en LCR. En la forma tardía, los síntomas aparecen a partir de la primera semana de vida. Existe frecuentemente meningitis y rara vez septicemia. La transmisión sería a través del canal del parto o en forma horizontal.

La forma de presentación habitual en la mujer embarazada es el síndrome febril sin foco (bacteriemia) (65\% de los casos); otras formas de presentación son: síndrome gripal $(30 \%)$, dolor abdominal $(20 \%)$ y cefalea $(10 \%)^{8}$. La mayoría de las pacientes presenta el embarazo como el único factor predisponente; sin embargo, existen reportes en que co-existe una enfermedad inmunosupresora. Estos casos tienden a presentar evoluciones más graves. Se ha descrito muerte materna por listeriosis asociada a lupus y a infección por VIH/ SIDA $^{33,34}$. En nuestra experiencia, una paciente era portadora de colitis ulcerosa, en tratamiento corticoesteroidal. Ella presentó meningitis. Se diagnosticó y trató precozmente, evolucionando en forma satisfactoria.

El éxito de la terapia antimicrobiana está influenciado por el particular comportamiento de L. monocytogenes. Como se mencionó anteriormente, es una bacteria intracelular facultativa. El antimicrobiano deberá llegar al interior de la célula hospedera para actuar. La segunda dificultad es que, frecuentemente, compromete el SNC y/o al feto. El antimicrobiano, entonces, deberá sortear una segunda dificultad: atravesar las barreras hemato-encefálica y/o placentaria. Listeria monocytogenes es sensible in vitro a varios antimicrobianos. Sin embargo, presenta resistencia natural a cefalosporinas de tercera generación, fosfomicina y quinolonas. La experiencia clínica muestra que amoxicilina o ampicilina, en dosis altas, son los fármacos de elección, tanto en madres como RNs. Su asociación con gentamicina ejerce un efecto sinérgico ${ }^{35}$. Nuestras cuatro pacientes fueron tratadas con ampicilina, tres de ellas en asociación con gentamicina. En los laborato- 
rios de microbiología clínica no se realiza de rutina el test de susceptibilidad in vitro a antimicrobianos por no estar definidos los puntos de corte ${ }^{36}$.

Existe evidencia que el tratamiento de la listeriosis, sin interrumpir el embarazo, puede resultar exitoso desde el punto de vista perinatal, evitando las complicaciones graves de la prematurez cuando este cuadro se presenta en edades gestacionales muy precoces ${ }^{37}$. Se ha prolongado desde una a veinte semanas la gestación desde el momento del diagnóstico de la infección ${ }^{38}$.

Finalmente, el médico deberá tener en consideración:

- El aspecto preventivo. Educar a la mujer gestante para que evite comer carne cruda o mal cocida, lavar cuidadosamente frutas y verduras, no consumir leche no pasteurizada, evitar consumo de quesos blandos y comidas rápidas ${ }^{37}$.

- Tener alta sospecha de listeriosis frente al síndrome febril en la mujer embarazada, recordando la alta positividad de los hemocultivos $(65 \%)$ en este cuadro, y la utilidad de la amniocentesis para el diagnóstico de infección intra-amniótica.

- Utilizar amoxicilina o ampicilina, en dosis altas, como terapia de elección, pudiendo asociar gentamicina por su efecto sinérgico.

- En casos de prematuros extremos, se ha reportado el tratamiento antimicrobiano exitoso sin interrupción del embarazo. Es una alternativa que debe ser discutida en casos seleccionados.

\section{Resumen}

Listeria monocytogenes es un bacilo grampositivo, intracelular facultativo, que se encuentra ampliamente difundido en la naturaleza, frecuentemente en alimentos. Las infecciones afectan principalmente a pacientes inmunocomprometidos, ancianos, mujeres embarazadas y neonatos. La infección intrauterina puede producir importantes complicaciones como corioamnionitis, parto de pre-término, aborto espontáneo de primer o segundo trimestre, mortinatos y sepsis neonatal. En el período 2001-2005, 16 pacientes con infección por L. monocytogenes fueron identificados en nuestro hospital. Cuatro de ellos (25\%) se presentaron en mujeres embarazadas; se describen sus características clínicas y de laboratorio. Hubo tres partos de pretérmino y un aborto espontáneo de segundo trimestre. En tres de las cuatro pacientes, el único factor de riesgo fue el embarazo. Una paciente recibía terapia inmunosupresora por una colitis ulcerosa. Fiebre fue el síntoma más frecuente. El compromiso feto-neonatal se manifestó por listeriosis neonatal precoz (dos casos) y mortinato (un caso). El embarazo puede ser el único factor predisponente a desarrollar listeriosis. Ésta debe considerarse en la evaluación del síndrome febril de una mujer embarazada. Los cultivos de sangre y líquido amniótico son útiles para su diagnóstico. La tasa de complicaciones perinatales permanece elevada.

\section{Referencias}

1.- Gellin B G, Broome C V. Listeriosis. JAMA 1989; 261: 1313-20.

2.- Gerner-Smidt P, Ethelberg S, Schiellerup P, Christensen J J, Engberg J, Fussing V, et al. Invasive listeriosis in Denmark 1994-2003: a review of 299 cases with special emphasis on risk factors and mortality. Clin Microbiol Infect 2005; 11: 618-24.

3.- Malik S V S, Barbuddhe S B, Chaudhari S P. Listeric infections in humans and animals in the Indian subcontinent: a review. Trop Anim Health Pro 2002; 34: 359-81.

4.- Mascola L, Sorvillo F, Goulet V, Hall B, Weaber R, Linnan M. Fecal carriage of Listeria monocytogenes- observations during a community- wide, common- source outbreak. Clin Infect Dis 1992; 15: 557-8.

5.- Doganay M. Listeriosis: clinical presentation. FEMS Immunol Med Microbiol 2003; 35: 173-5.

6.- Yildiz O, Aygen B, Esel D, Kayabas U, Alp E, Sumerkan B, et al. Sepsis and meningitis due to Listeria monocytogenes. Yonsei Med J 2007; 48: 433-9.
7.- Antal E A, Dietrichs E, Loberg E M, Melby KK, Maehlen J. Brain stem encephalitis in listeriosis. Scan J Infect Dis 2005; 37: 190-4.

8.- Mylonakis E, Paliou M, Omán E, Calderwood S, Wing E. Listeriosis during pregnancy. A case series and review of 222 cases. Medicine 2002; 81: 260-8.

9.- Silver H M. Listeriosis during pregnancy. Obstet Gynecol Surv 1998; 53: 737-40.

10.- Sirry H W, George R H, Whittle M J. Meningo-encephalitis due to Listeria monocytogenes in pregnancy. $\mathrm{Br} \mathrm{J}$ Obstet Gynaecol 1994; 101: 1083-4.

11.- Boucher M, Yonekura M L. Listeria meningitis during pregnancy. Am J Perinatol 1984; 1: 312-8.

12.- Antal E A, Høgåsen H R, Sandvik L, Maehlen J. Listeriosis in Norway 19772003. Scan J Infect Dis 2007; 39: 398-404.

13.- Bittencourt A L, García A G. Pathogenesis and pathology of hematogenous infections of the fetus and newborn. Pediatr Pathol Mol Med 2002; 21: 353-99.

14.- Ramaswamy V, Cresence V M, Rejitha J S, Lekshmi M U, Dharsana K S, Prasad S P, et al. Listeria- review of epidemiology and pathogenesis. J Microbiol Immunol Infect 2007; 40: 4-13.

15.- Posfay-Barbe K M, Wald E R. Listeriosis. Pediatr Rev 2004; 25: 151-9.

16.- Hjaltested E K, Gudmundsdóttir S, Jónsdóttir K, Kristinsson $\mathrm{K}$ G, Steingrímsson $\mathrm{O}$, Kristjánsson M. Listeriosis in Iceland, 1978-2000: A description of cases and molecular epidemiology. Scand J Infect Dis 2002; 34: 735-41.

17.- Doorduyn $\mathrm{Y}$, de Jager C M, van der Zwaluw W K, Wannet W J, van der Ende A, Spanjaard L, et al. Invasive Listeria monocytogenes infections in the Netherlands, 1995-2003. Eur J Clin Microbiol Infect Dis 2006; 25: 433-42.

18.- Liu D. Identification, subtyping and virulence determination of Listeria monocytogenes, an important foodborne pathogen. J Med Microbiol 2006; 55: 645-59.

19.- Sleator R D, Gahan C G, Hill C. A postgenomic appraisal of osmotolerance in Listeria monocytogenes. Appl Environ Microbiol 2003; 69: 1-9. 
20.- Lecuit M. Understanding how Listeria monocytogenes targets and crosses host barriers. Clin Microbiol Infect 2005; 11: 430-6.

21.- Buchholz U, Mascola L. Transmission, pathogenesis, and epidemiology of Listeria monocytogenes. Infect Dis Clin Pract 2001; 10: 34-41.

22.- Pinner R W, Schuchat A, Swaminathan B, Hayes P S, Deaver K A, Weaver R E, et al. Role of foods in sporadic listeriosis. II. Microbiologic and epidemiologic investigation. The Listeria Study Group. JAMA 1992; 267: 2046-50.

23.- Schlech W F 3rd, Lavigne P M, Bortolussi R A, Allen A C, Haldane E V, Wort A J, et al. Epidemic listeriosisevidence for transmission by food. N Engl J Med 1983; 308: 203-6.

24.- Linnan M J, Mascola L, Lou X D, Goulet V, May S, Salminen C, et al. Epidemic listeriosis associated with Mexican-style cheese. N Engl J Med 1988; 319: 823-8. 25.- Centers for Disease Control and Prevention
(CDC). Multistate outbreak of listeriosisUnited States, 2000. MMWR Morb Mortal Wkly Rep 2000; 49: 1129-30.

26.- Schlech W F 3rd. Foodborne listeriosis. Clin Infect Dis 2000; 31: 770-5.

27.- Antal E A, Høgåsen H R, Sandvik L, Maehlen J. Listeriosis in Norway 19772003. Scan J Infect Dis 2007; 39: 398-404.

28.- Siegman-Igra Y, Levin R, Weinberger M, Golan Y, Schwartz D, Samra Z, et al. Listeria monocytogenes infection in Israel and review of cases worldwide. Emerg Infect Dis 2002; 8: 305-10.

29.- Goulet V, Jacques C, Martin P, Vaillant V, Laurent E, de Valk H. Surveillance of human listeriosis in France, 2001-2003. Euro Surveill 2006; 11: 79-81.

30.- Cisternas A, Lagos N, Galstuch J, González C, García C, Díaz J. Infección por Listeria monocytogenes y embarazo con buen resultado perinatal. Rev Chil Obstet Ginecol 2002; 67: 237-41.

31.- Zelada M, Navarrete C. Infección neonatal por Listeria monocytogenes. Rev Chil
Pediatr 1990; 61: 17-24.

32.- Lobos T, Silva E, Maldonado A, Valenzuela M, Arancibia E, Montecinos D. Listeriosis perinatal. Rev Chil Infect 1984; 2: 101-3.

33. - Fan Yuan-Da, Pastorek J, Janney A, Sanders C. Listeriosis as an obstetric complication in an immunocompromised patient. South Med J 1989; 82: 1044-5.

34.- Wetli C, Roldan E, Fojaco R. Listeriosis as a cause of maternal death: an obstetric complication of the acquired immunodeficiency syndrome. Am J Obstet Gynecol 1983; 147: 7-9.

35.- Hof H. Therapeutic options. FEMS Imm Med Microbiol 2003: 203-5.

36. - CLSI. 2007. Performance standards for antimicrobial susceptibility test. Seventeenth Informatical Supplement.

37. - Abarca K. Infecciones en la mujer embarazadas transmisibles al feto. Rev Chil Infect 2003; 20 (supl 1): s41-s46.

38. - Katz V, Weinstein L. Antepartum treatment of Listeria monocytogenes septicemia. South Med J 1982; 75: 1353-4. 